\title{
A Smart Sensors-based real-time decision making system for the Cold Supply Chain
}

\author{
Jinlong Luo, a , Jianjun $\mathrm{Yi}^{1, \mathrm{~b}}$, Xiaomin $\mathrm{Zhu}^{1, \mathrm{c}}$ and Jun $\mathrm{Xu}^{1, \mathrm{~d}}$ \\ ${ }^{1}$ Department of Mechanical Engineering, East China University of Science and Technology, \\ Shanghai 200237, China

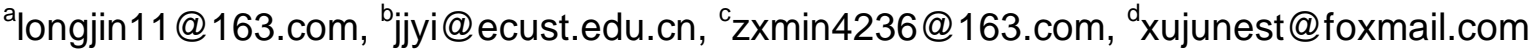

Keywords: Smart sensor; Intelligent Decision support System; Dynamic weight Dijkstra algorithm; Cold Chain Logistis

Abstract. Real-time monitoring of the cold chain logistics and timely decision-making are important means to ensure the quality of the cold supply chain. Therefore, this paper design a smart temperature and humidity tag with ad-hoc network, which can realize regular monitoring of temperature and humidity inside the cold chain logistics vehicles. Besides, based on the smart sensor this paper proposes a decision making system can real time acquire vehicle location information and make the necessary decisions on vehicle routing. According to the transportation time, temperature and humidity the system can predict the quality change trend of cold supply chain products. Finally, experiments show that the intelligent decision system proposed in this paper can effectively manage the cold chain products and provide the effective basis for the safety of cold chain products, thereby it can reduce the waste of the cold chain products and the economic loss of the cold chain logistics.

\section{Introduction}

A distinguished characteristics of all products for cold supply chains is their degradation in quality over time. The rate of degradation depends on the initial quality of the product as well as the ambient conditions they are subjected to until they reach the end of the supply chain. Obviously, a higher initial quality and relatively slow rate degradation would result in a better quality product. While reasonable steps are taken to ensure their quality, it is rather challenging to accomplish this due to the inherent limitations in currently used technology both in warehouses as well as during transportation. A related issue is that of contamination and how tracking and tracing of foods helps control the spread of contaminated items. The use of the smart sensors in perishable food cold chains for tracking or tracing and also to improve the level of control ambient conditions and thereby the quality of the perishable item[1].

Smart sensor technology has been under rapid development in recent years. According to the above analysis this paper puts forward a kind of smart temperature and humidity tag. Compared with the traditional digital temperature and humidity sensor, the smart temperature and humidity tag has the following advantages: First, the smart temperature and humidity tag embedded ZigBee transceiver module, which possesses ad-hoc network function, so it improves the flexibility and scalability of the construction of monitoring system;Second,the smart temperature and humidity tag with intelligent decision support function, according to the requirements of cold chain logistics and combined with improved Dijkstra route decision algorithm makes the vehicle intelligent decision system with strong function of prediction and decision-making, and helps the staff to enhance the decision-making level and the quality of the products; Finally, the smart temperature and humidity tag with the low power consumption property, which greatly reduces the energy consumption and significantly improving the service life of the tag.

\section{Design of the Smart Temperature and Humidity Tag}

This paper adopts embedded technology to develop a smart temperature and humidity tag, which is mainly responsible for obtaining temperature and humidity data and realizing communication 
between each node through the built-in transmission module. In order to ensure the stability and reliability of the tag, the structure of the smart tag designed is shown in fig.1(a), which is composed of the following five modules: CC2530 module, temperature and humidity sensor module, flash module, ZigBee data transmission module and power module.

After repeated demonstration, this paper selects TI CC2530 as the main control chip. CC2530 combines the good performance of RF transceiver, which is an excellent SoC solution, and its main functions are: (1). realizing timing acquisition of temperature and humidity; (2). transmit the data through the RF transceiver; (3). receive host instruction through the I/O port. In addition, this paper select the SHT11 digital temperature and humidity sensor launched by Scnsirion in Switzerland, which has the characteristics of high integration, simple interface, high precision and small size. So it is widely used in HVAC, automotive, consumer electronics, automatic control and other fields.Besides, The Flash is used to storage some parameters of the tags.

In this paper, the proposed smart temperature and humidity tag is mainly used for monitoring the temperature and humidity of the cold chain logistics vehicles. According to the actual situation of temperature and humidity changes inside the cold chain logistics car, the temperature and humidity resampling frequency will be set ten seconds. It would more real reaction temperature and humidity change trend without long time to keep the sensor node in working state. The smart tag proposed in this paper shown in the fig.1(b).

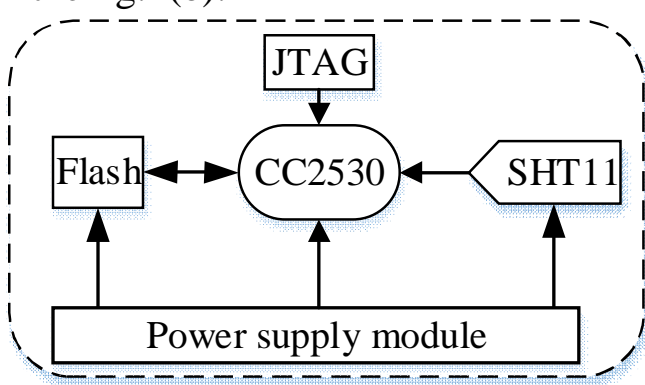

(a)

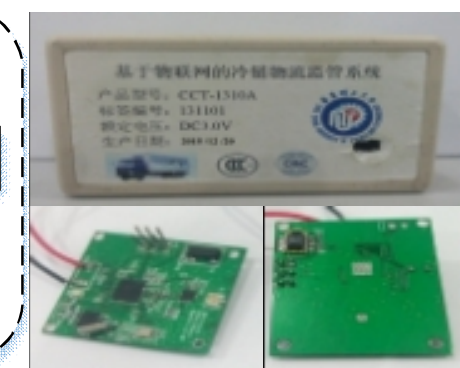

(b)

Fig.1 The structure of the smart sensor \& smart temperature and humidity tag

\section{Intelligent decision support system for cold chain logistics vehicle}

Cold supply chain management can keep freshness and safety of products untill the products reached consumers[2]. According to the smart temperature-humidity tag proposed above, a vehicular intelligent decision system proposed in this paper. The vehicular intelligent decision support system mentioned in this paper mainly involves intelligent decision administrator, wireless sensor networks based on smart sensors and decision-making executive. First of all, the intelligent decision-administrator formulate the systematic reasoning and decision rules according to actual demand and embeds it into smart sensor. Secondly, since the sensor with the function of ad-hoc network, so according to actual demand to reasonably layout of sensors and quickly build wireless sensor network. Finally, the system will real-time monitor information of cold chain logistics compartments' temperature and humidity, vehicle location and so on when the system works normally. Besides, according to the alarm information makes the most reasonable decision and inform decision-making implementer to take appropriate measures to reduce the economic loss in the process of the cold chain transportation. The structure of the system is shown in fig. 2 .

According to the actual requirements, the quality of the product in the cold chain logistics is directly influenced by transportation time.In addition, the compartment environment parameter (temperature \& humidity) in cold chain logistics vehicle also have a greater impact on the quality of cold chain products[3], $Q_{0}$ is the initial quality of the product. Supposing ignore some other minor factors, transportation time and temperature and humidity parameters can be used to forecast the quality of cold chain products according the following Eq.1: 
(1) $\beta$ shows sensitivity coefficient of perishable food to time. Firstly, different perishable products have different sensitivity coefficient to time. Secondly, for the same product in different storage temperature and humidity environment can also affect products' sensitive coefficient to time. Therefore, different values should be determined according to transportation products and storage environment[4].

(2) $t$ shows transportation time. Therefore, the quality of cold supply chain products can be predicted according to the Eq. 1.

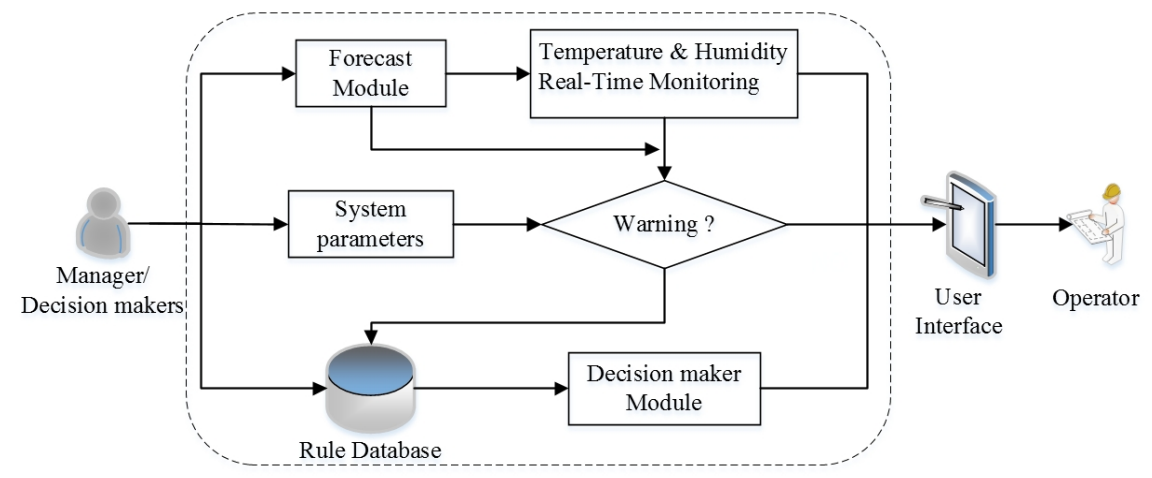

Fig. 2 The structure of the system

\section{Case Study}

According to the actual demand of cold chain logistics, this paper fully considers the feature of products and reasonably layout smart sensors in the cold chain logistics compartment[5], as shown in fig.3(b). The intelligent decision terminal placed in cold chain logistics vehicle cab which can help cold chain logistics decision-making executive improve the decision-making level and quality and reduce the wastage of the cold chain products at any time. The intelligent decision terminal includes MCU, ZigBee coordinator, Flash, GPS/GPRS module, alarm device, LCD module and power module, as shown in fig.3(a). Through the ZigBee coordinator, the intelligent decision terminal can read temperature and humidity in each node of compartment regularly and timing obtain information of vehicle location, weather and road conditions by GPS/GPRS module. The MCU makes comprehensive decision according to above information, ultimately converts this information into transport time, expected arrival time, environmental information (temperature and humidity information) to comprehensive description the quality of cold chain products, and finally predicts the current quality $Q_{t}$ and final quality $Q_{f}$ of cold chain products, and then let the information regularly display on the LCD. Cold chain logistics workers will make corresponding operation according to above decision information.

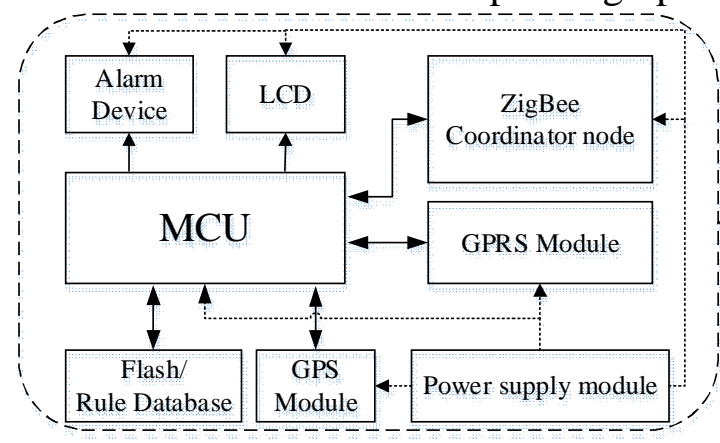

(a)

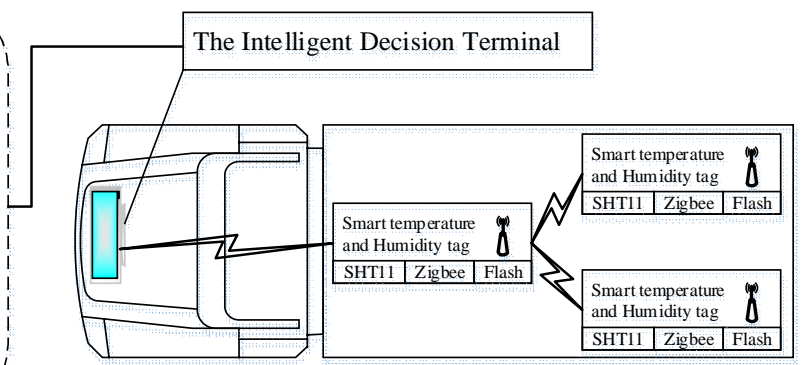

Cold chain logistics vehicle

(b)

Fig. 3 The layout of the smart sensors in the cold chain logistics vehicle

It is obviously that choice of path directly reflects transport time and finally affects quality of the cold supply chain products. Thus, decision of transport path is very important and necessary. Based on Dijkstra's algorithm this paper can solve the shortest path problem between origin and destination. 
Suppose fig.4(a) is a city path layout and there is a constant " $w$ " called weight in every path. It's basic principle is: From the starting point every time a new points is extended with the small weight. Finally, the sum of weight of the travel route is minimum in all routes. Weight value is determined by a lot of factors, such as road quality and twists and turns degree of path. In addition, The historical traffic data and weather factors also affect the weight in this path.

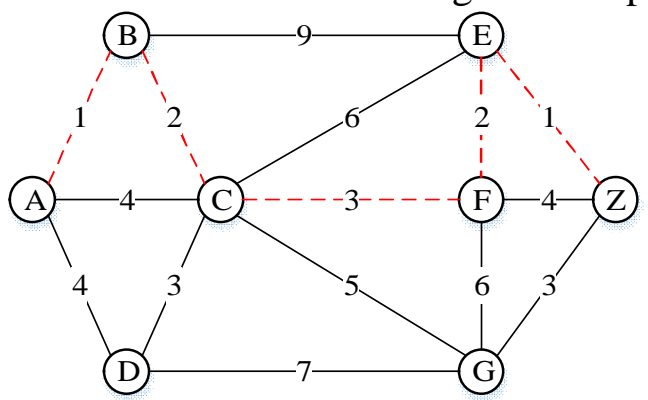

(a)

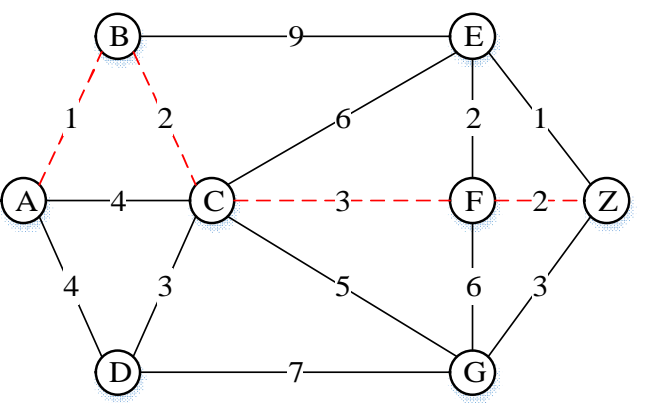

(b)

Fig.4 (a) Initial path decision result; (b) Path decision result after weight change

Dijkstra's algorithm can ensure the shortest path from point A to Z. This path shown in fig.4(a) will be as the original plan for this transport. However, the weight value of Dijkstra algorithm is a fixed constant, can be called single weight, which does not match with the actual traffic situation. Generally speaking, the geospatial data shows the uncertainty, and in the process of actual transportation the weight value may be a variable[6]. Due to many factors affect the weight value on each path, the weight value of Dijkstra algorithm on each path can be regarded as a single weight which get from different factors according to matching degree. Table 1 lists some factors that affect the weights on each path, according to the actual situation each factor will be given an initial value, the single weight can be calculated by Eq.2.Therefore, it's necessary to re-plan transport path in the process of each transport intersection according to the actual conditions. Suppose starting point A to $\mathrm{Z}$ according to the original plan, vehicular terminal receives traffic jam information during the path from $\mathrm{C}$ to $\mathrm{F}$ when reaching $\mathrm{C}$. It's necessary to put $\mathrm{C}$ as starting point and $\mathrm{Z}$ as ending point re-plan new path according to Dijkstra algorithm. When the car will reach to $\mathrm{C}$ the system will modify weight value from $\mathrm{C}$ to F.Finally, the re-planned path is shown as fig.4(b):

Table 1. Factors affect route weight

\begin{tabular}{ccc}
\hline Weight & Influence factors & Correlation \\
\hline$w_{1}$ & Path distance & $x_{1}$ \\
$w_{2}$ & Road conditions & $x_{2}$ \\
$w_{3}$ & Weather factor & $x_{3}$ \\
$w_{4}$ & Driver factor & $x_{4}$ \\
$\ldots$ & $\ldots$ & $\ldots$ \\
$w_{n}$ & Transport tool factor & $x_{n}$ \\
\hline
\end{tabular}

$$
w=\sum_{i=1}^{n} x_{i} w_{i}
$$

In summary, this paper comprehensively predicts the quality of cold chain products through transport time and environment parameters $(\mathrm{T} \& \mathrm{H})$. Firstly, temperature and humidity value obtained on time in the carriages of cold chain logistics determines reduced rate of product quality with time goes on. Secondly, Vehicle location information can predict current quality $Q_{t}$ and final quality $Q_{f}$ to destination on time[7]. $Q_{q}$ is the lowest quality of the product that consumer can accept.The workflow chart of decision System is shown in fig.5. 


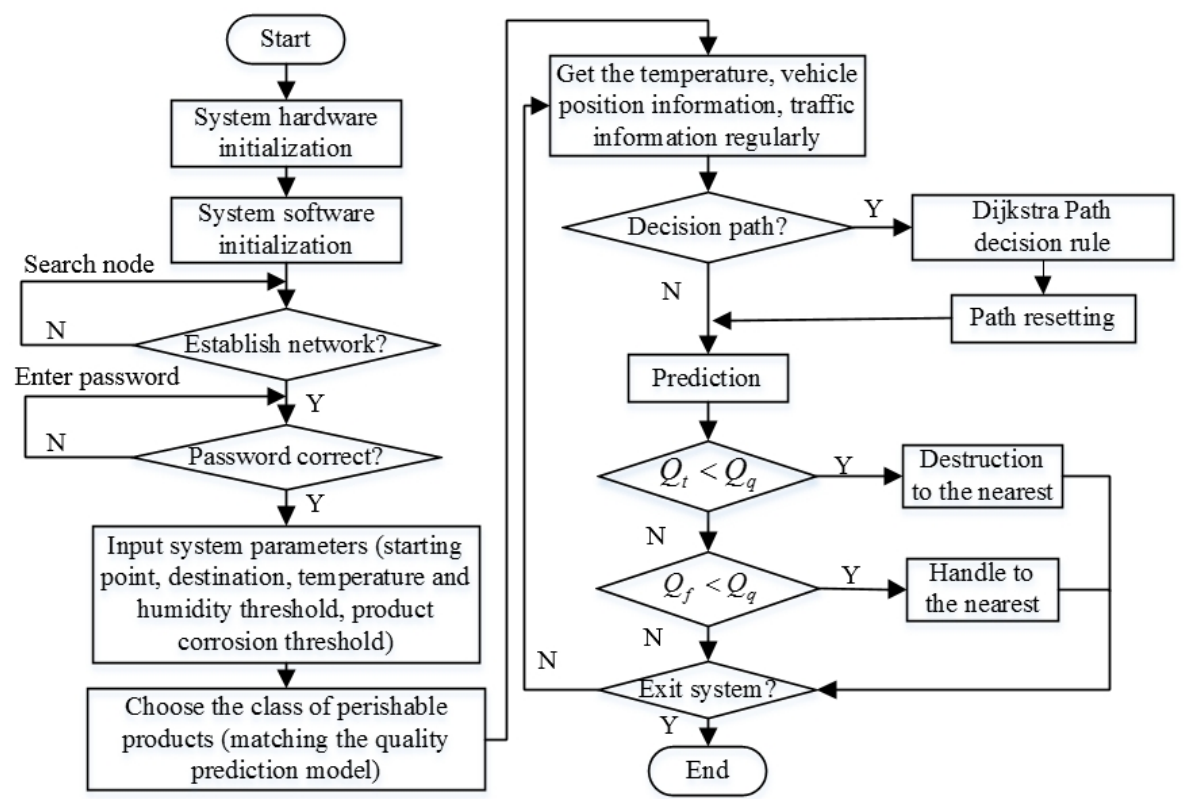

Fig. 5 The flow chart of System software and Emergency Treatment

According to the function requirements of the proposed system, the following several groups of experimental data tested and compared to show the performance of the system. In order to consider the feasibility of test, this research takes the transport of lettuce from East China University of Science and Technology to Tongji University as an example. According to actual condition, path information model can be simplified as shown in the fig. 6 .

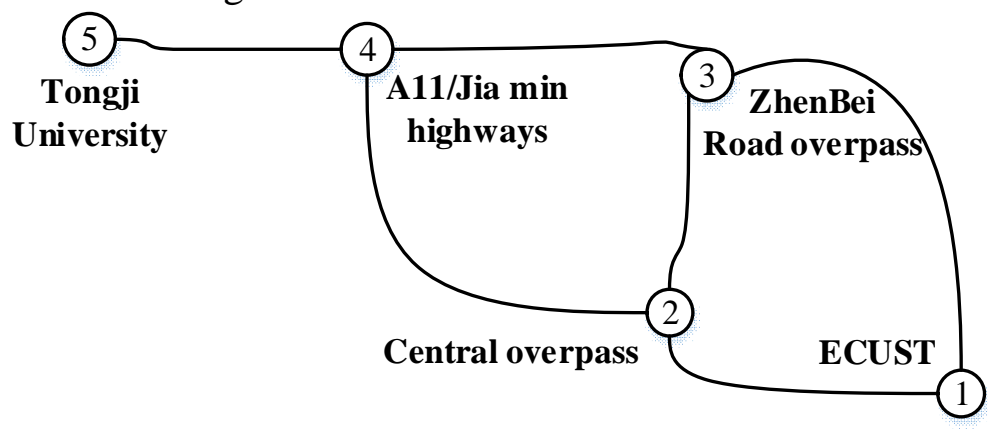

Fig. 6 The actual simplified model

Table 2. Monitoring Parameter Values and path decision result

\begin{tabular}{l|lllll}
\hline \multicolumn{1}{c}{ Parameter } & \multicolumn{1}{c}{$\mathbf{1}^{\text {st }}$ Group } & \multicolumn{1}{c}{$\mathbf{2}^{\text {st }}$ Group } & \multicolumn{1}{c}{$\mathbf{3}^{\text {st }}$ Group } & $\mathbf{4}^{\text {st }}$ Group & \multicolumn{1}{c}{$\mathbf{5}^{\text {st }}$ Group } \\
\hline Temperature & 0.50 & 0.50 & 0.50 & 0.50 & 0.50 \\
Humidity & $90 \%$ & $90 \%$ & $90 \%$ & $90 \%$ & $90 \%$ \\
Start & ECUST & ECUST & ECUST & ECUST & ECUST \\
End & Tongji & Tongji & Tongji & Tongji & Tongji \\
Start Time & $15 / 10 / 608: 09$ & $15 / 10 / 608: 09$ & $15 / 10 / 708: 00$ & $15 / 10 / 713: 00$ & $15 / 10 / 718: 00$ \\
Product Name & Lettuce & Lettuce & Lettuce & Lettuce & Lettuce \\
Number & 20 & 20 & 20 & 20 & 20 \\
End Time & $15 / 10 / 609: 40$ & $15 / 10 / 609: 55$ & $15 / 10 / 709: 25$ & $15 / 10 / 714: 09$ & $15 / 10 / 719: 05$ \\
Conveyance & Refrigerator & Refrigerator & Refrigerator & Refrigerator & Refrigerator \\
& car & car & car & car & car \\
Route & $1-2-4-5$ & $1-3-4-5$ & $1-2-4-5$ & $1-2-3-4-5$ & $1-2-4-5$ \\
$Q_{0}$ & $95 \%$ & $95 \%$ & $95 \%$ & $95 \%$ & $95 \%$ \\
$Q_{f}$ & $90 \%$ & $91 \%$ & $93 \%$ & $93 \%$ & $94 \%$ \\
\hline
\end{tabular}

In order to verify and evaluate the performance of the system, several groups of experiments were carried out in different situations, the experimental data is shown in table 2 . Under the condition of 
without using the system, the vehicle operation routes is different because of the experience and the hobby of drivers are different. The first set of experiment and the second group experiment show this kind of phenomenon. The following three groups of experiments illustrate three different cases using the system, according to the actual weight of each section the system makes comprehensive decision and provides the most optimized path. Therefore, the concrete experimental analysis shows this system has good performance in real-time path decisions, at the same time, it can effectively help staff to prevent degradation and decline in the value of the product in the cold chain products transportation and effectively reduce the loss of products in the process of transportation. Because of the transport time is very short, so the performance of the system is not obvious. With the increase of transportation time, the effect of the system will be more obvious.

\section{Conclusions}

According to the actual requirement of cold chain logistics, this paper puts forward a smart temperature and humidity tag with ad-hoc network function and a smart sensors-based real-time decision making system. Experiments show that the intelligent decision-making system has a certain role in the management of cold chain logistics products. Besides, the smart temperature and humidity tag also can be used in every situation that needs real-time monitoring of temperature and humidity parameters because of its small volume, high precision, high stability and ad-hoc network function.

\section{Acknowledgements}

This paper is supported by the Research Foundation of Science and Technology Commission of Shanghai under Grant no. 10DZ1500200, the Nature Science Fund of China (NSFC) under Grant nos. 50975088, 51275173, and 51575186, the Fundamental Research Funds for the Central Universities under Grant no. WH0913009, Shanghai Pujiang Program under Grant no. PJ201000353, and Shanghai Software and IC industry Development Special Fund under Grant no. 120493.

\section{References}

[1] Lin Qi; Mark Xu; Zetian Fu; Trebar Mira; Xiaoshuan Zhang. A WSN-based perishable food shelf-life prediction and LSFO strategy decision support system in cold chain logistics. Food Control 2014, 19-29.

[2] E. Abad; F. Palacio; M. Nuin; A. González de Zárate; A. Juarros; J.M. Gómez; S. Marco. RFID smart tag for traceability and cold chain monitoring of foods: Demonstration in an intercontinental fresh fish logistic chain. Journal of Food Engineering 2009, 394-399.

[3] Lixing Wang; S.K. Kwok; W.H. Ip. A radio frequency identification and sensor-based system for the transportation of food. Journal of Food Engineering 2010, 120-129.

[4] BAO Hai-rong; ZHANG Kui. Effect of different refrigeration temperatures on quality changes of fresh salmon. Science and Technology of Food Imdustry 2012, 33, 344-347

[5] Liu Chen; Zhang Ruirui; Zhao Chunjiang. Design of Wireless Sensor Node Used for Agricultural Products Cold Chain Logistics Environment Information Collection Based on ZigBee. Journal of Agricultural Mechanization Research 2014, 6, 198-201.

[6] CHEN Xiao-hong; WANG Yan-juan. Realization of Dynamic Route Guidance Algorithm Based Improved Dijkstra. Science Technology and Engineering 2008, 8, 6024-6027.

[7] E. Gogou ; G. Katsaros ; E. Derens ; G. Alvarez ; P.S. Taoukis . Cold chain database development and application as a tool for the cold chain management and food quality evaluation. International Journal of Refrigeration 2015, 52 , 109-121. 